ACR30/50/70/90 responses in the anakinra group were sustained throughout the study period. Patients in the anakinra group had a prompt and persistent decrease in CRP and ferritin levels at Week 1, which was not observed in the placebo group. There were no unexpected safety findings. All anakinra patients developed anti-drug antibodies (ADAs) at some timepoint during the study. ADAs were persistent throughout the treatment period, except in one patient. Titers were low to moderate. One placebo patient had low ADA titers at one occasion. No neutralizing antibodies were observed and the ADAs did not appear to impact clinical efficacy or safety.

Conclusion: Anakinra is superior to placebo in the treatment of Still's disease. ADAs occur frequently but do not appear to adversely impact efficacy or safety. These results confirm the benefits of anakinra treatment in patients with active, newly diagnosed Still's disease across ages.

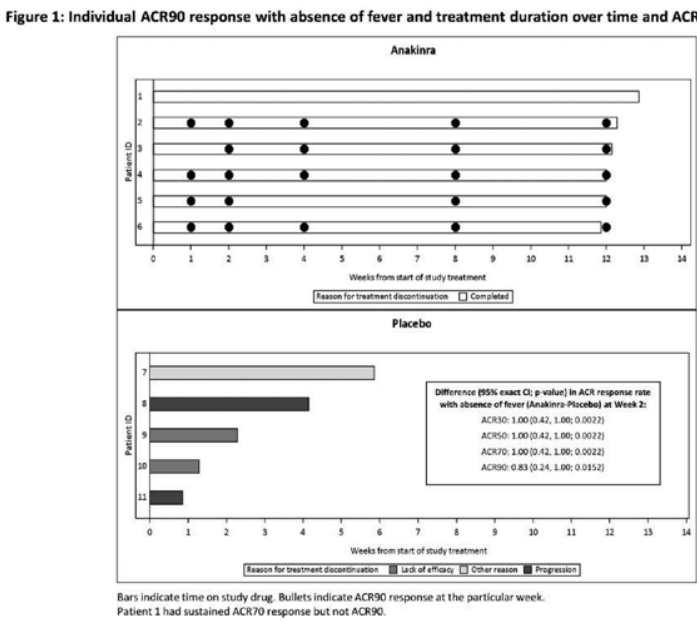

Figure 2: Time to study drug discontinuation, Kaplan-Meier plot

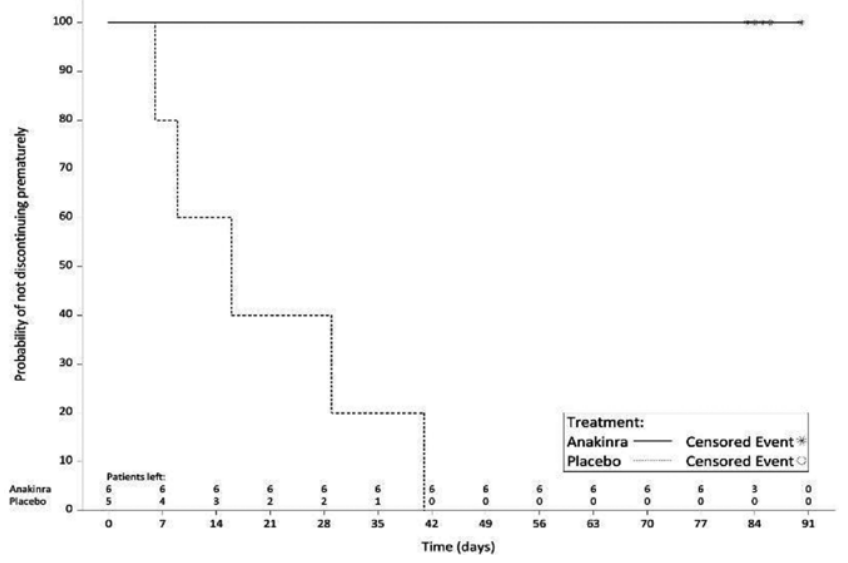

Disclosure of Interests: Laura Schanberg Grant/research support from: Sobi, BMS, Consultant of: Aurinia, UCB, Sanofi, Peter Nigrovic Grant/research support from: Novartis, BMS, Pfizer, Consultant of: Novartis, BMS, Pfizer, Sobi, Miach Orthopedics, Simcere, XBiotech, Quench Bio, Ashley Cooper: None declared, Winn Chatham Grant/research support from: Sobi, Consultant of: Sobi, Shoghik Akoghlanian: None declared, Namrata Singh: None declared, Egla Rabinovich Grant/research support from: AbbVie, UCB Pharma, Janssen Research \& Development, Akaluck Thatayatikom: None declared, Alysha Taxter: None declared, Jonathan Hausmann Consultant of: Novartis, Milan Zdravkovic Shareholder of: Sobi, Employee of: Sobi, Sven Ohlman Shareholder of: Sobi, Employee of: Former employee of Sobi, Henrik Andersson Employee of: Sobi, Susanna Cederholm Shareholder of: Sobi, Employee of: Sobi, Margareta Wikén Shareholder of: Sobi, Employee of: Former employee of Sobi, Rayfel Schneider Grant/research support from: Roche, Novartis, Sobi, Pfizer, Consultant of: Sobi, Novartis, Novimmune, Fabrizio De Benedetti Grant/research support from: AbbVie, Pfizer, Novartis, Novimmune, Sobi, Sanofi, Roche, Speakers bureau: AbbVie, Novartis, Roche, Sobi

DOI: 10.1136/annrheumdis-2020-eular.2822

\section{AB1060 A RARE IGG4-REALTED DISEASE PHENOTYPE WITH BONE DESTRUCTIVE LESIONS}

E. Sokol ${ }^{1}$, S. Palshina ${ }^{1} .{ }^{1}$ V.A. Nasonova Research Institute of Rheumatology, Moscow, Russian Federation

Background: IgG4-related disease (IgG4-RD) is an immunomediated fibroinflammatory condition with systemic course that can affect almost any organ in the body. In the majority of cases it is a benign condition with slow progression. The clinical symptoms are usually determined by compression of nearby anatomic structures by tumefective lesions, but not by invasive growth of the pseudotumor with the destruction of the organs. Bone destruction always raises suspicion of malignant tumor, but it should be considered in the course of IgG4-RD as well.

Objectives: To report a very rare bone destructive phenotype of IgG4-RD.

Methods: We report 5 cases of biopsy proven IgG4-RD with bone destructive lesions.

Results: In our cohort of patients there were 3 patients with multiple fascial bones destruction due to maxillary sinus pseudotumor expansion and 2 patient with vertebral destruction lesions.

Patients 1, 2 and 3 with facial bone destruction were young men aged 42, 36 and 28 years. In all cases the primary lesion was located in the maxillary sinus with expansion to the fascial soft tissues and orbit. Two patients had probable and 1 possible IgG4-RD diagnosis according to consensus diagnostic criteria, 2011. All patients were treated with rituximab and low doses of glucocorticoids with improvement of clinical symptoms (fascial edema, eye pain and/or headache), but lacking in radiologic improvement.

Patient 4 is a 51-year-old man who developed retrosternal pain, mimicking angina pectoris. Chest CT showed a tumor of posterior mediastinum, infiltrating the right lung and Th6-Th8 bodies destruction. Biopsy of the lesion revealed lymphoplasmatic infiltrate with lymph follicles formation, few eosinophils, significant diffuse fibrosis. Immunohistochemical study, showed IgG:IgG4 ratio $>40 \%$. The serum IgG4 concentration was $1.94 \mathrm{~g} / \mathrm{l}$ (normal range below $2.0 \mathrm{~g} / \mathrm{l}$ ). Combined treatment was administered: rituximab $500 \mathrm{mg}$ weekly \#4 and cyclophosphamide $1000 \mathrm{mg}+$ methylprednisolone $250 \mathrm{mg}$ IV every 2 weeks \#6. After that - cyclophosphamide $200 \mathrm{mg}$ per week intramuscularly, methylprednisolone $4 \mathrm{mg}$ daily per os. Due to spinal instability the patient undergone surgery. Six months later at check-up examination CT has shown a dramatic decrease of the mediastinal infiltrate. The treatment with cyclophosphamide and oral metylprednisolone was tapered gradually during 2 years due to no sites of pathological hypermetabolic activity were found on PET.

Patient 5 is a 60-year-old woman who had spinal surgery for $\mathrm{C} 2$ odontoid destruction. Biopsy revealed chronic inflammatory with massive fibrosis. 1,5 years later she developed salivary glands enlargement. During evaluation she had elevated serum IgG4 4.9 g/l and IgM 4.1 g/l (normal range below 0.6-3.7 g/l), serum protein electrophoresis with immunofixation showed monoclonal IgMк $3.2 \mathrm{~g} / \mathrm{l}$. Left submandibular salivary gland biopsy revealed significant fibrosis, dense lymphoplasmatic infiltrate forming lymph follicle with IgG:IgG4 ratio $50-80 \%$ and no pathological signs of lymphoma. The patient was treated with rituximab $500 \mathrm{mg}$ per week \#2 and cyclophosphamide $1000 \mathrm{mg}+$ methylprednisolone $250 \mathrm{mg}$ IV every 2 weeks \#3. The treatment was discontinued because of toxic hepatitis. Unfortunately, no follow-up was available. The tissue specimens after spinal surgery were also unavailable.

Conclusion: IgG4-RD can manifest by bone, including vertebral, destructive lesions and thus should be included when considering differential diagnosis in patients with bone destruction.

Disclosure of Interests: None declared

DOI: 10.1136/annrheumdis-2020-eular.6391

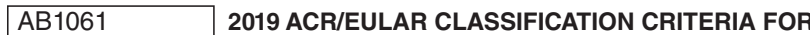 IGG4-RELATED DISEASE IN RUSSIAN COHORT OF PATIENTS.}

E. Sokol ${ }^{1}$, S. Palshina ${ }^{1}$, A. Torgashina ${ }^{1}$, J. Khvan ${ }^{1} .{ }^{1}$ V.A. Nasonova Research Institute of Rheumatology, Moscow, Russian Federation

Background: IgG4-related disease (IgG4-RD) is a systemic immunomediated fibroinflammatory condition that can affect almost any organ in the body. This is the reason for dramatic variety of clinical symptoms and complexity of diag nostics. 2011 Comprehensive diagnostic criteria (CDC) for IgG4-RD are used to establish the diagnosis for all lesions (except autoimmune pancreatitis type 1). In 2019 the new ACR/EULAR classification criteria for IgG4-RD were proposed to facilitate the formation of more homogenous groups of patients primarily for clinical trials inclusion purpose. They also provide a framework for clinicians considering diagnosis of IgG4-RD.

Objectives: To evaluate 2019 ACR/EULAR classification criteria for IgG4-RD in Russian cohort of patients with IgG4-RD. 